TRANSACTIONS OF THE

AMERICAN MATHEMATICAL SOCIETY

Volume 357, Number 9 , Pages 3553-3566

S 0002-9947(04)03594-9

Article electronically published on December 9, 2004

\title{
SMOOTH PROJECTIVE VARIETIES WITH EXTREMAL OR NEXT TO EXTREMAL CURVILINEAR SECANT SUBSPACES
}

\author{
SIJONG KWAK
}

\begin{abstract}
We intend to give a classification of smooth nondegenerate projective varieties admitting extremal or next to extremal curvilinear secant subspaces. Gruson, Lazarsfeld and Peskine classified all projective integral curves with extremal secant lines. On the other hand, if a locally Cohen-Macaulay variety $X^{n} \subset \mathbb{P}^{n+e}$ of degree $d$ meets with a linear subspace $L$ of dimension $\beta$ at finite points, then length $(X \cap L) \leq d-e+\beta$ as a finite scheme. A linear subspace $L$ for which the above length attains maximal possible value is called an extremal secant subspace and such $L$ for which length $(X \cap L)=d-e+\beta-1$ is called a next to extremal secant subspace.

In this paper, we show that if a smooth variety $X$ of degree $d \geq 6$ has extremal or next to extremal curvilinear secant subspaces, then it is either Del Pezzo or a scroll over a curve of genus $g \leq 1$. This generalizes the results of Gruson, Lazarsfeld and Peskine (1983) for curves and the work of M-A. Bertin (2002) who classified smooth higher dimensional varieties with extremal secant lines. This is also motivated and closely related to establishing an upper bound for the Castelnuovo-Mumford regularity and giving a classification of the varieties on the boundary.
\end{abstract}

\section{§0. Introduction}

Let $X$ be a nondegenerate smooth projective variety of dimension $n$, degree $d$ and codimension $e$ in $\mathbb{P}^{n+e}$ defined over an algebraically closed field $k$ of characteristic zero. Consider a line $\ell$ in the Grassmannian $\mathbb{G}(1, n+e)$ which meets with $X$ at finitely many points. What is the maximal length of the scheme $X \cap \ell$ ? It is well known and can be proved by an elementary argument (cf. [GLP]) that

$$
\text { length }(X \cap \ell) \leq d-e+1 \text {. }
$$

When $e=1$, i.e., $X$ is a hypersurface, the equality is always true by the simple Bezout theorem. A line $\ell$ for which the above length attains maximal possible value is called extremal and such an $\ell$ for which length $(X \cap \ell)=d-e$ is called a next to extremal secant line to $X$. More generally, if $X \cap L$ is a finite scheme for a nondegenerate, locally Cohen-Macaulay variety $X$ and a linear subspace $L$ of dimension $\beta, 1 \leq \beta \leq e$ in $\mathbb{P}^{n+e}$, then it can be shown (see [K3]) that

$$
\text { length }(X \cap L):=\operatorname{dim}_{k}\left(\mathcal{O}_{\mathbb{P}^{n+e}} / \mathcal{I}_{X}+\mathcal{I}_{L}\right) \leq d-e+\beta \text {. }
$$

Received by the editors July 3, 2003 and, in revised form, December 3, 2003.

2000 Mathematics Subject Classification. Primary 14M07, 14N05, 14J30.

This work was supported by grant No. R02-2001-000-00004 from the Korea Science and Engineering Foundation (KOSEF). 
As in the case of secant lines, we can define extremal (resp. next to extremal) secant subspace $L$ to $X$ if length $(X \cap L)=d-e+\beta$ (resp. $d-e+\beta-1$ ). We should remark here that the above upper bound is not true in general in case $X$ is not locally Cohen-Macaulay. Of special importance are extremal secant and next to extremal secant lines. An obvious classical observation is that a variety $X$ having an $m$-secant line cannot be defined by equations of degree less than $m$. A stronger and more modern version of this claim is that $X$ cannot be $(m-1)$-regular. More precisely, Castelnuovo-Mumford regularity can be defined generally for a coherent sheaf $\mathcal{M}$ on $\mathbb{P}^{n+e}$ as follows: $\mathcal{M}$ is said to be $m$-regular if $H^{i}\left(\mathbb{P}^{n+e}, \mathcal{M}(m-i)\right)=0$ for all $i>0$ and $\operatorname{reg} \mathcal{M}=\min \{m \in \mathbb{Z}: \mathcal{M}$ is $m$-regular $\}$. For a projective scheme $X$ with the ideal sheaf $\mathcal{I}_{X}, \operatorname{reg}(X)$ is defined to be reg $\left(\mathcal{I}_{X}\right)$. In particular, it gives information on the existence of maximal possible multisecant lines to a projective variety $X$ (see Proposition 1.1(b)). For a nondegenerate projective curve $C$, it was proved by Castelnuovo and Gruson-Lazarsfeld-Peskine that reg $(C) \leq d-e+1$ and if $\operatorname{reg}(C)=d-e+1$, then $C$ is a smooth rational curve having a $(d-e+1)$ secant line with a couple of easy exceptions (cf. [GLP]). Precisely speaking, let $C \subset \mathbb{P}^{n}$ be a nondegenerate reduced irreducible projective curve of degree $d \geq n+2$ with an extremal secant line. Then there are two types of surjective morphisms of $\mathcal{O}_{\mathbb{P}^{1}}$-module, i.e.

(a) $\mathcal{O}_{\mathbb{P}^{1}}(1) \oplus \mathcal{O}_{\mathbb{P}^{1}}(n-2) \longrightarrow \mathcal{O}_{\mathbb{P}^{1}}(d) \longrightarrow 0$,

(b) $\mathcal{O}_{\mathbb{P}^{1}} \oplus \mathcal{O}_{\mathbb{P}^{1}} \oplus \mathcal{O}_{\mathbb{P}^{1}}(n-2) \longrightarrow \mathcal{O}_{\mathbb{P}^{1}}(d) \longrightarrow 0$,

and their tautological projective embedding of projective bundles gives smooth rational curves of degree $d$ with the unique extremal $(d-e+1)$-secant line which are clearly contained in rational scrolls. For further results about bounding regularity of curves, see $[\mathrm{D}]$ and $[\mathrm{No}]$. Note that A. Noma obtained a regularity bound including the arithmetic genus of curves for the first time. For higher-dimensional cases, it was recently proved in $[\mathrm{Be}]$ that a projective variety of dimension $\geq 2$ with an extremal secant line is one of the following:

(a) a cone over the Veronese surface in $\mathbb{P}^{5}$;

(b) a cone over a projected Veronese surface in $\mathbb{P}^{4}$;

(c) a cone over a smooth rational scroll $\Sigma$.

Of course, these varieties play an important role in the following regularity problems due to Castelnuovo, Mumford, Eisenbud and Goto (EG], GLP]):

(a) $\operatorname{reg}(X) \leq d-e+1$;

(b) classify all extremal and next to extremal varieties.

Note that if $X$ has an $m$-secant line, then $\operatorname{reg}(X) \geq m$ (see Proposition 1.1). M-A. Bertin showed in her thesis that $\operatorname{reg}(X)=d-e+1$ for all projective varieties having a $(d-e+1)$-secant line, which gives further evidence to the above regularity conjecture (a).

However, for higher-dimensional projective varieties, it is widely open to get the sharp regularity bound and the classification of boundary examples even in the case of smooth varieties ([K1]). With respect to the above problems, it would also be very interesting to classify projective varieties with extremal or next to extremal secant lines or secant subspaces which give not only some information about the geometric structure of those special varieties but also boundary examples of the Castelnuovo-Mumford regularity conjecture. 
In the present paper, we are intended to classify smooth projective varieties having next to extremal secant lines and more generally having extremal or next to extremal curvilinear secant subspaces, thus extending the work of M-A.Bertin [Be]. Roughly speaking, these varieties are either scrolls over a curve of genus $g \leq 1$ or Del Pezzo varieties. These varieties are also special from the adjunction theoretic point of view. In particular, if we assume that a given projective variety $X$ is embedded by the complete linear system and has no extremal curvilinear secant subspaces, then we have the following (Corollary 4.4): a smooth projective variety $X^{n} \subset \mathbb{P}^{n+e}, n \geq 1, e \geq 3$, has a next to extremal secant line if and only if $X$ is one of the following:

(a) $X$ is either an elliptic normal curve or an elliptic surface scroll $\mathbb{P}(\mathcal{E})$ embedded by the complete linear system $\left|C_{0}+b f\right|, \operatorname{deg}(b)=-c_{1}(\mathcal{E})+3$, where $\mathcal{E}$ is a normalized rank two vector bundle and $C_{0}$ is a minimal section.

(b) $X$ is a Del Pezzo variety.

Note that a smooth projective variety admitting an extremal curvilinear secant subspace is always rational. In addition, as Professor Fyodor Zak suggested, we give some interesting examples which have next to extremal curvilinear secant subspaces of dimension $\geq 2$ but no next to extremal secant lines (Proposition 4.5 and Example 4.6).

\section{$\S 1$. Preliminaries}

Throughout this paper we work over an algebraically closed field $k$ of characteristic zero. In this section we recall basic results which will be used in what follows.

Proposition 1.1. Suppose that a coherent sheaf $\mathcal{M}$ on $\mathbb{P}^{n+e}$ is m-regular. Then $\mathcal{M}$ has the following properties:

(a) $\mathcal{M}(m)$ is generated by its sections;

(b) the multiplication map $H^{0}\left(\mathcal{O}_{\mathbb{P}^{n+e}}(l)\right) \otimes H^{0}(\mathcal{M}(m)) \rightarrow H^{0}(\mathcal{M}(m+l))$ is surjective for $l \geq 0$.

Proof. See $[\mathrm{Mu}]$.

If $\ell$ is an $(m+1)$-secant line of $X$, then, by Proposition $1.1(\mathrm{~b}), X$ cannot be $m$-regular. Therefore, $X$ can always be cut out by equations of degree at most $\operatorname{reg}(X)$. On the other hand, for smooth projective varieties of codimension two, one has the following result:

Theorem 1.2. Let $X$ be a smooth threefold of degree $d$ in $\mathbb{P}^{5}$ which is not contained in any quadric hypersurface. Then, $\operatorname{reg}(X) \leq d-3$. Furthermore, $\operatorname{reg}(S) \leq d-3$ for any regular surface $S \subset \mathbb{P}^{4}$ with $H^{0}\left(\mathcal{I}_{S}(2)\right)=0$ with the exception of the projected Veronese surface.

Proof. See [K2], Theorem 2.11.

Theorem 1.3. Let $X$ be a locally Cohen-Macaulay codimension 2 subvariety of dimension $n \geq 2$. If $X$ is contained in a quadric hypersurface, then $X$ is one of the following:

(a) $X$ is a complete intersection of two hypersurfaces of degrees 2 and $m$ and $\operatorname{reg}(X)=m+1$; 
(b) $X$ is arithmetically Cohen-Macaulay of degree $2 m-1$ which is defined by three equations of degrees $2, m$ and $m$ and $\operatorname{reg}(X)=m$.

Proof. This is elementary, and we also remark that $X$ is not necessary smooth; for details, see [K2].

Definition 1.4. Let $X^{n}$ be a nondegenerate smooth variety of degree $d$ in $\mathbb{P}^{n+e}$ and let $1 \leq \beta \leq e-1$.

(a) A linear subspace $L$ of dimension $\beta$ with length $(X \cap L)=d-e+\beta$ (resp. $d-e+\beta-1$ ) is called an extremal (resp. next to extremal) secant subspace to $X$.

(b) If length $(X \cap L)$ is finite and $\mathcal{O}_{X \cap L, q} \simeq k[t] /\left(t^{m}\right)$ for some $m>0$, i.e. $\operatorname{dim}\left(T_{q}(X) \cap L\right) \leq 1$ for all $q \in \operatorname{Supp}(X \cap L)$, then $L$ is called a curvilinear secant subspace to $X$.

The following proposition and corollary seem to be well known. However, for lack of suitable reference, we give elementary proofs here.

Proposition 1.5. Let $X^{n} \subset \mathbb{P}^{n+e}$ be a nondegenerate integral variety and let $L=\mathbb{P}^{\beta}, 1 \leq \beta \leq e-1$, be a multisecant subspace to $X$. Then, for a general linear subspace $M$ such that $L \subset M=\mathbb{P}^{m} \subset \mathbb{P}^{n+e}, \beta \leq m \leq e, X \cap M$ is also finite.

Proof. First assume that $L$ is a line. Then, for a general plane $M=\mathbb{P}^{2}$ such that $L \subset M \subset \mathbb{P}^{n+e}, X \cap M$ is also finite. Indeed, choose a general point $p \in L$ and consider the projection $\pi_{p}: \mathbb{P}^{n+e} \rightarrow \mathbb{P}^{n+e-1}$ from the point $p$. Since $p \notin X$, we have the finite morphism $\left.\pi_{p}\right|_{X}: X \rightarrow Y=\pi_{p}(X) \subset \mathbb{P}^{n+e-1}$. It is easy to show that $Y$ is irreducible and nondegenerate. Note that $\ell=\pi_{p}(M)$ is a general line through the point $q_{0}=\pi_{p}(L)$ which meets $Y$ at finitely many points $\left\{q_{0}, q_{1}, q_{2}, \cdots, q_{t}\right\}$ with some multiplicities. It is clear that $\operatorname{Supp}(X \cap M)=\operatorname{Supp}\left(\left.\pi_{p}\right|_{X}{ }^{-1}(\ell \cap Y)\right)$ as sets. Since $\left.\pi_{p}\right|_{X}$ is finite, $X \cap M$ is also a finite scheme.

For the general case, we use induction on $\operatorname{dim}(L)$ and $\operatorname{dim}(M)$. That is, choose a general point $p \in L \backslash X$ and consider the projection $\left.\pi_{p}\right|_{X}: X \rightarrow Y=\pi_{p}(X) \subset$ $\mathbb{P}^{n+e-1}$. Note that $M^{\prime}=\pi_{p}(M)$ is a general linear space containing $L^{\prime}=\pi_{p}(L)$ and $\operatorname{dim}\left(L^{\prime}\right)=\operatorname{dim}(L)-1, \operatorname{dim}\left(M^{\prime}\right)=\operatorname{dim}(M)-1$. By the induction hypothesis, $Y \cap M^{\prime}$ is a finite scheme. Then, by the above argument, $X \cap M$ is also a finite scheme.

Proposition 1.6. Let $X^{n} \subset \mathbb{P}^{n+e}$ be a nondegenerate smooth variety of degree $d$. If $L=\mathbb{P}^{\beta}, 1 \leq \beta \leq e$, is a curvilinear secant subspace of dimension $\beta$ to $X$, then length $(X \cap L) \leq d-e+\beta$.

Proof. Suppose length $(X \cap L) \geq d-e+\beta+1$. Since $L$ is curvilinear, for a general hyperplane $H$ containing $L, Y=X \cap H$ is also smooth and $L$ is a curvilinear secant to $Y$ of same length. Thus, we can pick up $(e-\beta)$ general points $p_{1}, p_{2}, \cdots, p_{e-\beta} \in$ $X \backslash L$ such that $M=\operatorname{Span}\left\langle L, p_{1}, p_{2}, \cdots, p_{e-\beta}\right\rangle \simeq \mathbb{P}^{e}$ is also curvilinear to $X$ and length $(X \cap M) \geq d+1$. Choose a linear subspace $M_{1} \simeq \mathbb{P}^{e-1}$ of $M$ which does not meet with $X$ and take a projection $\pi_{M_{1}}: X^{n} \longrightarrow \mathbb{P}^{n}$ with center $M_{1}$. Then $\pi_{M_{1}}: X^{n} \longrightarrow \mathbb{P}^{n}$ is a finite flat morphism (cf. Exercise 18.17, [E]) and consequently all fibers have the same length of degree $d$ which contradicts length $(X \cap M) \geq$ $d+1$. 
Corollary 1.7. Let $X^{n} \subset \mathbb{P}^{n+e}$ be a nondegenerate smooth variety of degree $d$. Suppose that $X$ has an extremal curvilinear secant subspace $L=\mathbb{P}^{\beta}$ for some $1 \leq$ $\beta \leq e-1$. Then $X$ is a rational variety.

Proof. Consider the projection $\pi_{L}: X \rightarrow \mathbb{P}^{n+e-\beta-1}$ with center at $L$. By the proof of Proposition 1.6, $\pi_{L}$ is generically one-to-one and thus $X$ is birationally equivalent to the closure of its image $Y=\pi_{L}(X)$ which is an irreducible and nondegenerate variety of minimal degree $e-\beta,[\mathrm{EH}]$. Thus $Y$ and hence $X$ is a rational variety.

Remark 1.8. The curvilinearity condition in Proposition 1.6 and Corollary 1.7 is used to show that the involved linear projections are generically one to one. However, for noncurvilinear cases, the projections cannot be even finite morphisms.

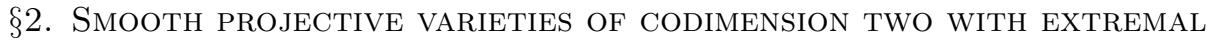 OR NEXT TO EXTREMAL SECANT LINES}

Lemma 2.1. Let $X^{n} \subset \mathbb{P}^{n+e}, n \geq 2$, be a smooth $n$-dimensional subvariety of degree $d$. If $X$ has a $m$-secant line $\ell$, then for a generic hyperplane $H$ containing $\ell, X \cap H$ is also smooth and $\ell$ is also $m$-secant to $X \cap H$.

Proof. First note that $X \cap H$ is smooth outside $X \cap \ell$ by Bertini's theorem. Let $\operatorname{Supp}(X \cap \ell)=\left\{p_{1}, p_{2}, \cdots, p_{t}\right\}$. Pick points $q_{i} \in \mathbb{T}_{p_{i}}(X) \backslash \ell$ where $\mathbb{T}_{p_{i}}(X)$ is the projective tangent space to $X$ at $p_{i}$. Then we can choose a generic hyperplane $H$ containing the line $\ell$, but not passing through the points $q_{i}, 1 \leq i \leq t$. By the Jacobian criterion for nonsingularity, $X \cap H$ is smooth at $p_{i}$ and, consequently, $X \cap H$ is smooth irreducible and $\ell$ is also $m$-secant to $X \cap H$.

Note that for a nondegenerate projective variety of degree $d$ and codimension two, any $(d-1)$-secant line is extremal. If $X$ is of low degree, viz. $d=3$ or 4 , then it is easy to consider whether such lines exist or not. Thus it is reasonable to assume $d \geq 5$. For a smooth space curve $C$ in $\mathbb{P}^{3}, C$ has an extremal secant line if and only if $C$ is contained in a smooth quadric and is of type $(1, d-1)$ or $(d-1,1)$ as a divisor (cf. e.g. GLP], §3). There are also many space curves of type $(2, d-2)$ of degree $d$ and genus $(d-3)$ with next to extremal secant lines. On the other hand, for a higher-dimensional codimension two smooth variety $X$ of degree $d \geq 6$, it can be shown that there are no extremal and next to extremal secant lines to $X$. The following proposition is a special case of Bertin's result $\mathrm{Be}$, but we give another proof based on regularity techniques which also yield Theorem 2.3.

Proposition 2.2. Let $X^{n} \subset \mathbb{P}^{n+2}$ be a smooth subvariety of dimension $n \geq 2$ and degree $d \geq 5$. Then $X$ does not have $(d-1)$-secant lines.

Proof. By Lemma 2.1, we can choose hyperplanes $H_{1}, \ldots, H_{n-2}$ so that $S=X \cap$ $H_{1} \cap H_{2} \cap \cdots \cap H_{n-2}$ is smooth and $\ell$ is a $(d-1)$-secant line of $S \subset \mathbb{P}^{4}$. By Corollary 1.7, $S$ is rational, hence regular.

Suppose first that $h^{0}\left(\mathcal{I}_{S}(2)\right)=0$. Then $\operatorname{reg}(S) \leq d-3$ by Theorem 1.2. Therefore, $S$ has no $(d-1)$-secant line by Proposition $1.1(\mathrm{~b})$.

Suppose now that $h^{0}\left(\mathcal{I}_{S}(2)\right) \neq 0$. Then, by Theorem 1.3, either $S=Q \cap Z(F)$ is a complete intersection of degree $2 m$ and $\operatorname{reg}(S)=m+1$ or $S=Q \cap Z\left(F_{1}\right) \cap Z\left(F_{2}\right)$ is a projectively Cohen-Macaulay variety of degree $2 m-1$ and $\operatorname{reg}(S)=m=$ $\operatorname{deg}\left(F_{1}\right)=\operatorname{deg}\left(F_{2}\right)$. From the inequality $d-1 \leq \operatorname{reg}(S)$ it follows that the only 
possible value of $m$ is $m=2$ and $S$ is either a complete intersection of two quadrics or an intersection of three quadrics. In both cases $d=3,4$, and so the proof is complete.

Thus it is natural to consider the next to extremal case, i.e. the case of smooth varieties of codimension two having a $(d-2)$-secant line. As above, for all varieties of degree less than 6 , we observe that it is easy to consider whether such lines exist or not. So it remains to consider the case $d \geq 6$.

Theorem 2.3. Let $X^{n} \subset \mathbb{P}^{n+2}$ be a smooth subvariety of dimension $n \geq 2$ and degree $d \geq 6$. Then $X$ does not have $(d-2)$-secant lines.

Proof. Let $\ell$ be a $(d-2)$-secant line of $X$. Suppose first that $n=\operatorname{dim}(X) \geq 3$. As above, one can cut $X$ by successive general hyperplanes containing $\ell$ to get a smooth irreducible surface $S=X \cap H_{1} \cap H_{2} \cap \cdots \cap H_{n-2} \subset \mathbb{P}^{4}$ for which $\ell$ is a $(d-2)$-secant line. Note that $h^{1}\left(\mathcal{O}_{S}\right)=0$ because $h^{1}\left(\mathcal{O}_{X}\right)=0$ for $n \geq 3$ (Barth Theorem).

By Theorem 1.2, $h^{0}\left(\mathcal{I}_{S}(2)\right)=0$ implies reg $(S) \leq d-3$ unless $S$ is a projected Veronese surface for which $d=4$. Thus in this case $S$ does not have $(d-2)$-secant line.

Suppose now that $h^{0}\left(\mathcal{I}_{S}(2)\right) \neq 0$. Then, by Theorem 1.3, $S=Q \cap Z(F)$ is either a complete intersection of degree $2 m, m=\operatorname{deg} F$ and $\operatorname{reg}(S)=m+1$, or $S=Q \cap Z\left(F_{1}\right) \cap Z\left(F_{2}\right)$ is a projectively Cohen-Macaulay surface of degree $2 m-1$, $\operatorname{deg} F_{1}=\operatorname{deg} F_{2}=m$ and $\operatorname{reg}(S)=m$. From the inequality $d-2 \leq \operatorname{reg}(S)$ it follows that the only possible values of $m$ are $m=2$ and $m=3$. Therefore, $S$ is either a complete intersection of two quadrics or a projectively Cohen-Macaulay surface of degree 3 or 5 (note that if $S$ is a complete intersection of a quadric and a cubic with $d=6, S$ does not have 4 -secant lines). Since we assume $d \geq 6$, this completes the proof of the theorem for $n=\operatorname{dim}(X) \geq 3$ (and also for regular surfaces).

Suppose now that $S \subset \mathbb{P}^{4}$ is a smooth irregular surface. Let $\ell$ be a $(d-2)$ secant line of $S$, and let $\pi_{\ell}: S \rightarrow \mathbb{P}^{2}$ be the projection with center at $\ell$. Since $\operatorname{deg} X=d$, this rational map is generically 2 to 1 . Furthermore, the set $\Sigma=\{y \in$ $\left.\mathbb{P}^{2} \mid \operatorname{dim}\left(\left.\pi_{\ell}\right|_{S}\right)^{-1}(y)=1\right\}$ is finite. Taking a general hyperplane section $C$ of $S$ containing $\ell$, we see that the projection $\pi_{\ell}: C=S \cap H \rightarrow \mathbb{P}^{1}$ is a double covering. Therefore $C$ is a hyperelliptic curve. We denote by $g \geq 1$ the genus of $C$. If $g=1$, then $S$ is either a Del Pezzo surface or a quintic elliptic scroll (cf. [I0]). If $g \geq 2$, then by the classification of smooth projective varieties with a hyperelliptic curve section (cf. see (1.1), (1.2), (1.3) and Theorem 3.1 in [SV]), $S$ is either an elliptic scroll or a conic bundle over $\mathbb{P}^{1}$. However, in both cases one has $d \leq 5$ (cf. [ES]). Thus smooth surfaces of degree $d \geq 6$ in $\mathbb{P}^{4}$ do not have $(d-2)$-secant lines in $\mathbb{P}^{4}$.

We would like to remark that codimension two smooth varieties of dimension $\geq 4$ should be a complete intersection due to Hartshorne's conjecture. So it would be interesting to show that there is no $(d-1-\delta)$-secant line to these varieties for smaller $\delta \geq 2$ because the degree of defining equations are much smaller than degree of such a variety.

The following problem is also of interest from the point of view of Castelnuovo regularity. 
Problem 2.4. Classify all smooth surfaces $S \subset \mathbb{P}^{4}$ of degree $d$ with $\operatorname{reg} S=d-1$. In particular, does a smooth surface $S$ of degree $d \geq 5$ with $\operatorname{reg}(S)=d-1$ have a $(d-1)$-secant line?

It should be noted that the analog of 2.4 for space curves is well known GLP].

\section{§3. Smooth projective VARIETIES of CODIMENSion $e \geq 3$ With EXtremal OR NEXT TO EXTREMAL CURVILINEAR SECANT SPACE}

We can consider extremal or next to extremal curvilinear secant subspaces as well as secant lines for smooth projective varieties of codimension $e \geq 3$. First of all, we proceed with giving classification of varieties of arbitrary codimension $e \geq 3$ with next to extremal secant lines thus extending M-A. Bertin's work Be. We start with the following observation. Let $X$ be a smooth $n$-dimensional variety of degree $d$ and codimension $e$. Suppose $X$ has an extremal $(d-e+1)$-secant line $\ell$ and that the projection $\pi_{p}: X \rightarrow \pi_{p}(X) \subset \mathbb{P}^{n+e-1}$ from a point $p$ outside of $\operatorname{Sec}(X)$ is an isomorphism. Then $\pi_{p}(\ell)$ is at least next to extremal secant to $\pi_{p}(X)$ because $\operatorname{deg} X=\operatorname{deg} \pi_{p}(X), \operatorname{codim}\left(X, \mathbb{P}^{n+e}\right)=\operatorname{codim}\left(\pi_{p}(X), \mathbb{P}^{n+e-1}\right)+1$ and

$$
\text { length }\left(\pi_{p}(X) \cap \pi_{p}(\ell)\right) \geq(d-e+1) .
$$

Theorem 3.1 (M-A. Bertin). Let $X^{n} \subset \mathbb{P}^{n+e}, n \geq 1, e \geq 2$, be a nondegenerate smooth variety of degree $d$, and let $\ell$ be an extremal secant line in $\mathbb{P}^{n+e}$ such that length $(X \cap \ell)=d-e+1$. Then, $X$ is one of the following:

(a) $X$ is the Veronese surface in $\mathbb{P}^{5}$ or a projected Veronese surface in $\mathbb{P}^{4}$;

(b) $X$ is a rational scroll.

Proof. Let $\ell$ be an extremal secant line to $X$, so that

$$
\text { length }(X \cap \ell)=d-e+1 \text {. }
$$

By Corollary 1.7 and Lemma 2.1, taking successive generic hyperplane sections containing the line $\ell$, we get a smooth rational curve $C$ for which $\ell$ is an extremal secant line. Thus the sectional genus of $X$ vanishes, i.e. $g(X, H)=0$. Hence $\Delta(X, H)=0$, where $\Delta(X, H)=d-h^{0}\left(X, \mathcal{O}_{X}(1)\right)+n$ is the $\Delta$-genus of $X$. We get the conclusion by the well-known classification of smooth varieties with $\Delta(X, H)=$ 0 (cf. e.g. $[\mathrm{Fu}]$ ).

Theorem 3.1 can be generalized to the case of extremal curvilinear secant subspaces to $X$ as follows.

Proposition 3.2. Let $X^{n} \subset \mathbb{P}^{n+e}, n \geq 1, e \geq 2$, be a nondegenerate smooth variety of degree $d$, and let $L=\mathbb{P}^{\beta}, 1 \leq \beta \leq e-1$, be an extremal curvilinear secant subspace to $X$. Then the conclusion of Theorem 3.1 holds, i.e. $X$ is one of the following:

(a) $X$ is the Veronese surface in $\mathbb{P}^{5}$ or a projected Veronese surface in $\mathbb{P}^{4}$;

(b) $X$ is a rational scroll.

Proof. Let $L$ be an extremal $\beta$-dimensional curvilinear secant subspace to $X$ such that length $(X \cap L)=d-e+\beta$. Since $X \cap L$ is curvilinear, arguing as in the proof of Lemma 2.1, we can take successive generic hyperplane sections containing $L$ and meeting transversely the tangent spaces at all the points of $X \cap L$ at each step. Consequently, we get a smooth curve which is rational by Corollary 1.7. The argument is completed as in the proof of Theorem 3.1. 
Remark 3.3. As Fyodor Zak pointed out, it would be interesting to find out smooth varieties with extremal noncurvilinear secant subspaces which are neither rational scrolls nor Veronese surfaces.

For a smooth projective variety $X^{n} \subset \mathbb{P}^{n+e}, n \geq 1, e \geq 3$, with a $(d-e)$ secant line $\ell$ and general points $p_{1}, p_{2}, \ldots, p_{\beta}, \beta \leq e-2$, of $X$, first note that $L^{\beta+1}=\left\langle\ell, p_{1}, p_{2}, \ldots, p_{\beta}\right\rangle$ is either an extremal or next to extremal curvilinear secant subspace containing $\ell$. If it is extremal, then by Proposition 3.2, $X$ is either the Veronese surface or a rational scroll.

Therefore, we proceed with describing the structure of smooth projective varieties of arbitrary codimension $e \geq 3$ with a next to extremal secant line $\ell$ such that $L^{\beta+1}=\left\langle\ell, p_{1}, p_{2}, \ldots, p_{\beta}\right\rangle$ is always a next to extremal secant subspace if $X \cap L^{\beta+1}$ is finite for points $p_{1}, p_{2}, \ldots, p_{\beta}$ of $X$ and $1 \leq \beta \leq e-2$.

Theorem 3.4. Let $X^{n} \subset \mathbb{P}^{n+e}, n \geq 1$, e $\geq 3$, be a smooth variety of degree $d \geq 6$ with $a(d-e)$-secant line $\ell$. Suppose that $L^{\beta+1}=\left\langle\ell, p_{1}, p_{2}, \ldots, p_{\beta}\right\rangle$ is always a next to extremal secant subspace for points $p_{1}, p_{2}, \ldots, p_{\beta}$ of $X$ and $1 \leq \beta \leq e-2$. Then, $X$ is either a Del Pezzo variety or an elliptic scroll.

Proof. As mentioned before, note that if $L^{\beta+1}=\left\langle\ell, p_{1}, p_{2}, \ldots, p_{\beta}\right\rangle$ is an extremal secant subspace for some points $p_{1}, p_{2}, \ldots, p_{\beta}$ to $X, \beta \leq e-2$, then $X$ is either the Veronese surface or a rational scroll. Now, let $\ell$ be a given next to extremal $(d-e)$-secant line to $X$. We pick general points $p_{1}, p_{2}, \ldots, p_{e-3}$ in $X$ and consider the linear span

$$
L^{e-2}=\left\langle\ell, p_{1}, p_{2}, \ldots, p_{e-3}\right\rangle
$$

By our assumption,

$$
\text { length }\left(X \cap L^{e-2}\right)=d-3,
$$

i.e. $L^{e-2}=\mathbb{P}^{e-2}$ is a next to extremal secant subspace to $X$.

Consider the projection $\pi_{L^{e-2}}: X \rightarrow \mathbb{P}^{n+1}$ with center at $L^{e-2}$. This rational map is clearly generically finite. If $\pi_{L^{e-2}}$ has a fiber of length 2 at some point $q \in \mathbb{P}^{n+1}$, then the linear span $\left\langle L^{e-2}, \pi_{L^{e-2}}{ }^{-1}(q)\right\rangle=\mathbb{P}^{e-1}$ is an extremal secant space containing $\ell$, contrary to our assumption. Therefore, the fibers of $\pi_{L^{e-2}}$ are either positive dimensional or reduce to one point.

Let $Y^{n}$ be the closure of $\pi_{L^{e-2}}(X)$ in $\mathbb{P}^{n+1}$, and let $Z=\left\{y \in Y \mid \operatorname{dim}\left(\pi_{L^{e-2}}\right)^{-1}(y)\right.$ $\geq 1\}$. By the above, $\pi_{L^{e-2}}$ is generically one-to-one, and consequently, $\operatorname{deg}(Y)=$ $d-(d-3)=3, \operatorname{codim}_{Y} Z \geq 2$.

Now, take $(n-1)$ successive general hyperplane sections of the hypersurface $Y$ in $\mathbb{P}^{n+1}$. Note that such hyperplane sections are in one-to-one correspondence with general hyperplane sections of $X$ containing $L^{e-2}$. Let $C$ be the hyperplane section curve of $X$ cut out by the corresponding hyperplanes in $\mathbb{P}^{n+e}$, and consider the restriction $\left.\pi_{L^{e-2}}\right|_{C}$. Since $\operatorname{codim}_{Y} Z \geq 2$, a general plane section of $Y$ is a nonsingular plane cubic (cf. [Ha], Theorem 14.9). Consequently, $\left.\pi_{L^{e-2}}\right|_{C}$ is actually an isomorphism between the curve $C$ and an elliptic curve, so that the sectional genus $g(X)$ of $X$ is equal to 1 . From the sectional genus formula $2 g(X)-2=$ $\left[K_{X}+(n-1) H\right] H^{n-1}$, we get $\left[K_{X}+(n-1) H\right] H^{n-1}=0$. Thus, either $K_{X}+(n-1) H$ is trivial, i.e. $K_{X}=-(n-1) H$ (in which case $X$ is a Del Pezzo variety) or $h^{0}\left(X, K_{X}+(n-1) H\right)=0$ (in which case, by adjunction theorem [Io, Theorem 1.4], $X$ is an elliptic scroll). 
Corollary 3.5. Let $X^{n} \subset \mathbb{P}^{n+e}, n \geq 1, e \geq 3$, be a smooth variety of degree $d \geq 6$. Suppose $X$ has a next to extremal curvilinear subspace $L^{\beta}, 1 \leq \beta \leq e-2$, i.e. length $\left(X \cap L^{\beta}\right)=d-e+\beta-1$. We have the following:

(a) If $X$ has an extremal subspace containing $L^{\beta}$, then $X$ is either a rational scroll or the Veronese;

(b) if $X$ has no extremal subspace containing $L^{\beta}$, then $X$ is either a Del Pezzo variety or an elliptic scroll.

Proof. Proceeding as in the proof of Theorem 3.4, we pick general points $p_{1}, p_{2}, \ldots$, $p_{e-\beta-2}$ in $X$. Then $L^{e-2}=\left\langle L^{\beta}, p_{1}, p_{2}, \ldots, p_{e-\beta-2}\right\rangle=\mathbb{P}^{e-2}$ is curvilinear and

$$
\operatorname{length}\left(X \cap\left\langle L^{\beta}, p_{1}, p_{2}, \ldots, p_{e-\beta-2}\right\rangle\right)=d-2 \text { or } d-3 \text {. }
$$

By considering the projection $\pi_{L^{e-2}}$, we can also use the above argument in Theorem 3.4 to get the sectional genus $g(X)=0$ in the case of (a) (resp. $g(X)=1$ in the case of (b)). Thus we are done.

Remark 3.6. (a) As in Remark 3.3, it would also be interesting to classify smooth varieties with a next to extremal noncurvilinear secant space which are neither Del Pezzo nor elliptic scrolls.

(b) In comparison with Corollary 3.5, we can consider a next to extremal curvilinear space $L^{\beta}$ of dimension $\beta=e-1$ to a smooth projective variety $X$ whose subspaces are not next to extremal at all. In this case, we cannot get the same upper bound of the sectional genus. Instead, it can be easily shown that $X$ has a hyperelliptic curve section. For the classification of projective varieties with hyperelliptic curve sections, see $(2.1)$, [SV]. For example, let $X=\mathbb{P}(\mathcal{E}) \rightarrow C$ be a projective bundle over a curve $C$ of genus 2 where $\mathcal{E}$ is a normalized indecomposable rank 2 vector bundle with $\operatorname{deg}\left(\wedge^{2} \mathcal{E}\right)=2$. Consider an embedding of $X$ in $\mathbb{P}^{5}$ by the very ample complete linear system $\left|C_{0}+3 f\right|$ where $C_{0}$ is a minimal section. Then, $\operatorname{deg}(X)=\left(C_{0}+3 f\right) \cdot\left(C_{0}+3 f\right)=8$ and a generic element $D$ in $\left|C_{0}+3 f-\pi^{*}\left(K_{C}\right)\right|$ is a smooth space curve of degree 6 and genus 2 by the simple computation. By Cayley's formula, $D$ has a unique 4 -secant line $\ell$ which also meets with $X$ only at 4 points (up to length) by Theorem 3.4. Now, take a projection $\pi_{\ell}: X \rightarrow Y \subset \mathbb{P}^{3}$ where $Y$ is the closure of the image under $\pi_{\ell}$. Then we can check that $Y$ is a quadric hypersurface and $\pi_{\ell}$ is generically 2:1 (cf. $\overline{\mathrm{ABB}}$ ). Consequently, for a generic point $q \in Y$, the linear $\operatorname{span}\left\langle\ell, \pi_{\ell}{ }^{-1}(q)\right\rangle=\mathbb{P}^{2}$ is a 6 -secant plane to $X$ which is a next to extremal curvilinear subspace.

\section{§4. Existence of extremal or neXt to eXtremal SECANT SUbSPaCes AND EXAMPLES}

In the previous section, we obtained necessary conditions for smooth projective varieties to have extremal or next to extremal curvilinear subspaces. Note that the Veronese surface in $\mathbb{P}^{5}$ or the projected Veronese in $\mathbb{P}^{4}$ have obviously extremal secant lines. Now, we investigate whether extremal or next to extremal secant lines or subspaces exist or not when $X$ is either a scroll over a curve of genus $g \leq 1$ or a Del Pezzo variety. First of all, we consider projective varieties which are embedded by the complete linear system.

Example 4.1 (Del Pezzo varieties). Let $X^{n}$ be a linearly normal Del Pezzo variety of degree $d$ in $\mathbb{P}^{n+e}$. Then $X$ has no extremal curvilinear secant subspace but a next to extremal subspace $L^{\beta}$ for all $1 \leq \beta \leq e-1$. Indeed, we have $\Delta(X, H)=$ 
$\operatorname{deg}(X)+n-h^{0}\left(\mathcal{O}_{X}(1)\right)=1$. So $d=e+2$, i.e. $X$ is of next to minimal degree. In addition, it can be easily checked that $\operatorname{reg}(X)=3$ and $X$ is cut out by quadrics. Therefore, $X$ has no trisecant lines. More generally, let $\left\{p_{1}, p_{2}, \ldots, p_{\beta}\right\}, \beta \leq e$, be a general collection of $\beta$ points of $X$, and put $L^{\beta-1}=\left\langle p_{1}, p_{2}, \ldots, p_{\beta}\right\rangle=\mathbb{P}^{\beta-1}$. By Corollary 3.5, $X$ does not have extremal curvilinear secant subspaces. Therefore, we have $d-e+(\beta-1)-1=\beta$ and

$$
X \cap\left\langle p_{1}, p_{2}, \ldots, p_{\beta}\right\rangle=\left\{p_{1}, p_{2}, \ldots, p_{\beta}\right\} .
$$

Here $L^{\beta-1}$ is a next to extremal curvilinear secant subspace to $X$.

Next, we consider an $n$-dimensional scroll $X$ of degree $d$ over a curve, not necessarily linearly normal, embedded in a projective space $\mathbb{P}^{n+e}$. First note that $\operatorname{codim}(X)=e \geq n-1$ since otherwise, by the Barth-Lefschetz theorem, the rank of the Picard group of $X$ would be equal to one, which is not the case for scrolls. Furthermore, $e=n-1$ if and only if $X$ is the Segre embedding of $\mathbb{P}^{1} \times \mathbb{P}^{n-1}$ in $\mathbb{P}^{2 n-1}[\mathrm{Oh}$.

According to [Be, Theorem 4], $\operatorname{reg}(X) \leq d-e+1$ for all scrolls over a curve (this is the conjectured bound for arbitrary varieties). However, in order to classify scrolls with next to extremal secant lines, it is useful to compute precisely the Castelnuovo-Mumford regularity of rational and elliptic scrolls. Let $\Delta(X, H)=$ $\operatorname{deg} X+n-h^{0}\left(\mathcal{O}_{X}(1)\right)$ be the $\Delta$-genus of $(X, H)$ where $H$ is the hyperplane section divisor. In the following lemma we put together some known facts that will be used in this section.

Lemma 4.2. Let $X^{n} \subset \mathbb{P}^{n+e}, n \geq 1$, be an $n$-dimensional elliptic scroll and let $H$ be its hyperplane section divisor. Then:

(a) $\Delta(X, H)=n$.

(b) If $X$ is embedded by a complete linear system, then $X$ is projectively normal.

Proof. For an elliptic normal curve, it is clearly projectively normal and by Riemann-Roch, $\Delta(X, H)=1$. For $n \geq 2$, let $(X, H)=\left(\mathbb{P}(\mathcal{E}), \mathcal{O}_{\mathbb{P}(\mathcal{E})}(1)\right)$ be a scroll over an elliptic curve $C$. By the Riemann-Roch theorem for vector bundles, $\chi(\mathcal{E})=$ $c_{1}(\mathcal{E})+\operatorname{rank}(\mathcal{E})(1-g)=c_{1}(\mathcal{E})$. Furthermore, $h^{1}(C, \mathcal{E})=h^{0}\left(C, \mathcal{E}^{*}\right)=0$ because a nonzero section $s$ of $\mathcal{E}^{*}$ defines a surjective morphism

$$
0 \rightarrow \mathcal{K} \rightarrow \mathcal{E} \rightarrow \mathcal{O}_{C}(-D) \rightarrow 0,
$$

where $\mathcal{K}$ is the kernel of $s^{*}$ and $D$ is the zero locus of $s$. Since $\mathcal{E}$ is ample, from the above exact sequence it follows that $\mathcal{O}_{C}(-D)$ is also ample, which is clearly impossible. Thus $\chi(\mathcal{E})=h^{0}(C, \mathcal{E})=c_{1}(\mathcal{E})$. Note also that $\operatorname{deg} X=c_{1}(\mathcal{E})$ for a scroll over a curve. Thus, $\operatorname{deg}(X)=h^{0}(C, \mathcal{E})=h^{0}\left(\mathcal{O}_{\mathbb{P}(\mathcal{E})}(1)\right)=h^{0}\left(\mathcal{O}_{X}(1)\right)$ and $\Delta(X, H)=\operatorname{deg} X+n-h^{0}\left(\mathcal{O}_{X}(1)\right)=n$. The proof of (b) can be found in $\mathrm{AB}$ and $[\mathrm{Bu}]$.

In general, for nonlinearly normal scrolls, it is important to find $m_{1}$ such that $h^{1}\left(\mathcal{I}_{X}(j)\right)=0$ for all $j \geq m_{1}$ in order to compute Castelnuovo-Mumford regularity, i.e. the hypersurface sections of degree $k$ is complete. In the case of linearly normal elliptic scrolls, we can compute $\operatorname{reg}(X)$ by the standard cohomological computation.

Proposition 4.3. Let $X^{n}, n \geq 1$, be an elliptic scroll of degree $d$ in $\mathbb{P}^{n+e}$ which is embedded by a complete linear system. Then, we have

(a) $\operatorname{reg}(X)=3$. 
(b) If $X$ has a next to extremal secant line, then it is either an elliptic normal curve or an elliptic surface scroll $\mathbb{P}(\mathcal{E})$ embedded by the complete linear system $\left|C_{0}+b f\right|, \operatorname{deg}(b)=-c_{1}(\mathcal{E})+3$, where $\mathcal{E}$ is a normalized rank two vector bundle and $C_{0}$ is a minimal section.

Proof. Note that a linearly normal elliptic scroll is projectively normal by Lemma 4.2. So, it is enough to show that

$$
H^{i}\left(X, \mathcal{O}_{X}(2-i)\right)=0, \quad i \geq 1
$$

Clearly, a linearly normal elliptic curve is not 2-regular but 3-regular. Suppose $n \geq 2$; one has $h^{i}\left(\mathcal{O}_{X}(2-i)\right)=h^{i}\left(\mathcal{O}_{\mathbb{P}(\mathcal{E})}(2-i)\right)=h^{i}\left(C, \operatorname{Sym}^{2-i}(\mathcal{E})\right)=0$ for $i \geq 2$. For $i=1, h^{1}\left(\mathcal{O}_{X}(1)\right)=h^{1}\left(\mathcal{O}_{\mathbb{P}(\mathcal{E})}(1)\right)=h^{1}(C, \mathcal{E})=0$ by the argument in the proof of the preceding lemma. Thus reg $(X)=3$ and in particular, the linearly normal elliptic scrolls do not have 4-secant lines. On the other hand, $d-e=n+1$ and if it has a $(d-e)$-secant line, then $n=1,2$. When $n=1, d-e=2$ and an elliptic normal curve is cut out by quadrics and clearly has a 2-secant line. For an elliptic surface scroll $\mathbb{P}(\mathcal{E})$ where $\mathcal{E}$ is a normalized rank two vector bundle and $C_{0}$ is a minimal section, it is known that $\left|C_{0}+b f\right|$ is very ample if and only if $\operatorname{deg}(b) \geq-c_{1}(\mathcal{E})+3$. In addition, if $\operatorname{deg}(b)>-c_{1}(\mathcal{E})+3$, then the embedded scroll $X$ is cut out by quadrics, $[\mathrm{Bu}, \mathrm{GP}$. So there is no 3 -secant line to $X$. However, the elliptic surface scroll embedded by $\left|C_{0}+b f\right|, \operatorname{deg}(b)=-c_{1}(\mathcal{E})+3$ has the plane cubic curve corresponding to $C_{0}$ because $C_{0} .\left(C_{0}+\left(-c_{1}(\mathcal{E})+3\right) f\right)=3, g\left(C_{0}\right)=1$. So, trisecant lines exist only for this elliptic scroll.

Corollary 4.4. Let $X^{n}$ be a linearly normal smooth variety in $\mathbb{P}^{n+e}, n \geq 1, e \geq 3$. Suppose $X$ has no extremal curvilinear secant subspace. $X$ has a next to extremal secant line if and only if it is one of the following:

(a) $X$ is an elliptic normal curve;

(b) $X$ is an elliptic surface scroll $\mathbb{P}(\mathcal{E})$ embedded by $\left|C_{0}+b f\right|, \operatorname{deg}(b)=-c_{1}(\mathcal{E})+$ 3 , where $\mathcal{E}$ is a normalized rank two vector bundle and $C_{0}$ is a minimal section;

(c) $X$ is a Del Pezzo variety.

Proof. This follows from Example 4.1 and Proposition 4.3.

On the other hand, as Fyodor Zak suggested, it would also be very interesting to investigate whether or not an elliptic scroll $X^{n}, n \geq 2$, has next to extremal curvilinear spaces of dimension $\geq 2$ instead of next to extremal secant lines. We characterize the linearly normal smooth projective varieties of dimension $\leq 3$ in terms of the existence of next to extremal curvilinear spaces when $e \geq 3$.

Proposition 4.5. Let $X^{n} \subset \mathbb{P}^{n+e}, e \geq 3$, be a linearly normal elliptic scroll of dimension $n=2,3$ of degree $d$. Then $X$ has a next to extremal curvilinear secant subspace $L^{\beta}$ for some $1 \leq \beta \leq e-1$.

Proof. For an elliptic surface scroll $X$, we suppose that $X$ does not have a trisecant line. So, $e=\operatorname{codim}(X) \geq 3, d=3+e$. Choose a bisecant line $\ell$ to $X$ and general points $p_{1}, p_{2}, \ldots, p_{e-3}$ in $X$. Then $L=\left\langle\ell, p_{1}, p_{2}, \ldots, p_{e-3}\right\rangle \simeq \mathbb{P}^{e-2}$ and length $\left(\left\langle\ell, p_{1}, p_{2}, \ldots, p_{e-3}\right\rangle \cap X\right)=e-1$ or $e$. If the length is $d-e+[(e-2)-$ $1]=d-3=e$, then $L$ is next to extremal and we are done. Suppose that length $\left\langle\ell, p_{1}, p_{2}, \ldots, p_{e-3}\right\rangle \cap X=e-1$ and consider the linear projection $\pi_{L}: X \rightarrow$ $X^{\prime} \subset \mathbb{P}^{3}$ with center at $L$. This rational morphism is generically $m: 1$, and from 
the equality $(e-1)+m \operatorname{deg} X^{\prime}=d=e+3$, it follows that either $m=2$, $\operatorname{deg} X^{\prime}=2$ or $m=1, \operatorname{deg} X^{\prime}=4$. If $m=2$, then the morphism $\pi_{L}$ has a fiber of length 2 at some point $q \in X^{\prime}$. Therefore, the linear $\operatorname{span}\left\langle L, \pi_{L}^{-1}(q)\right\rangle=\mathbb{P}^{e-1}$ is next to extremal. On the other hand, if $m=1, \operatorname{deg} X^{\prime}=4$, then $X^{\prime}$ has the sectional genus 3 which is false by Riemann-Hurwitz theorem.

For a linearly normal elliptic threefold scroll $X \subset \mathbb{P}^{d-1}$ of degree $d=e+$ 4 , if $X$ has a trisecant line $\ell$, then $\operatorname{length}\left(\left\langle\ell, p_{1}, p_{2}, \ldots, p_{e-3}\right\rangle \cap X\right)=e$ or $e+$ 1. By the same argument as above, we are done. Suppose $X$ has no trisecant line. Then choose a bisecant line $\ell$ to $X$ and general points $p_{1}, p_{2}, \ldots, p_{e-3}$ in $X$. So, length $\left\langle\ell, p_{1}, p_{2}, \ldots, p_{e-3}\right\rangle \cap X=e-1, e$ or $e+1$. We have only to check the case of length $\left(\left\langle\ell, p_{1}, p_{2}, \ldots, p_{e-3}\right\rangle \cap X\right)=e-1$. For the linear $\operatorname{span} L=$ $\left\langle\ell, p_{1}, p_{2}, \ldots, p_{e-3}\right\rangle \simeq \mathbb{P}^{e-2}$, consider the linear projection $\pi_{L}: X \rightarrow X^{\prime} \subset \mathbb{P}^{3}$ with center at $L$. From the equality $(e-1)+m \operatorname{deg} X^{\prime}=d=e+4$, it follows that either $m=1, \operatorname{deg} X^{\prime}=5$ or $m=5, \operatorname{deg} X^{\prime}=1$. Both cases are contrary to the assumption by the same argument.

We can construct an elliptic scroll admitting a next to extremal secant subspace which has no extremal secant line.

Example 4.6. Let $C$ be an elliptic curve. Consider the following decomposable normalized vector bundle $\mathcal{E}_{0}=\mathcal{E}_{1} \oplus \mathcal{L}$ of rank $r$ where $\mathcal{L}$ is a line bundle of degree $\leq 0$. By tensoring some power of a positive degree line bundle over $C$, we can assume that $\mathcal{E}_{0}$ is very ample, i.e., $\mathcal{O}_{\mathbb{P}_{C}\left(\mathcal{E}_{0}\right)}(1)$ is very ample. Note that $h^{0}\left(\mathcal{O}_{\mathbb{P}_{C}\left(\mathcal{E}_{0}\right)}(1)\right)=$ $h^{0}\left(C, \mathcal{E}_{0}\right)=h^{0}\left(C, \mathcal{E}_{1}\right)+h^{0}(C, \mathcal{L})$. Let $m_{1}=h^{0}\left(C, \mathcal{E}_{1}\right), m_{2}=h^{0}(C, \mathcal{L})$ and $\pi_{0}: X \simeq$ $\mathbb{P}_{C}\left(\mathcal{E}_{0}\right) \rightarrow C$ be an embedded scroll of degree $m_{1}+m_{2}$ in $\mathbb{P}^{m_{1}+m_{2}-1}$ by the linear system $\left|\mathcal{O}_{\mathbb{P}_{C}\left(\mathcal{E}_{0}\right)}(1)\right|$. On the other hand, let $\pi_{1}: Y \simeq \mathbb{P}_{C}\left(\mathcal{E}_{1}\right) \rightarrow C$ be an embedded scroll of degree $m_{1}$ in $\mathbb{P}^{m_{1}-1}$ by $\left|\mathcal{O}_{\mathbb{P}_{C}\left(\mathcal{E}_{1}\right)}(1)\right|$ and $\psi_{|\mathcal{L}|}(C)$ be an elliptic normal curve of degree $m_{2}$ in $\mathbb{P}^{m_{2}-1}$ embedded by the complete linear system $\psi_{|\mathcal{L}|}$. Note that $\mathbb{P}^{m_{1}-1}$ and $\mathbb{P}^{m_{2}-1}$ are complementary linear subspaces in $\mathbb{P}^{m_{1}+m_{2}-1}$. Thus $X$ is a scroll over an elliptic curve $C$ whose fiber is $\pi_{0}{ }^{-1}(p)=\left\langle\pi_{1}{ }^{-1}(p), \psi_{|\mathcal{L}|}(p)\right\rangle=\mathbb{P}^{r-1}$ for all $p \in C$, where $r=\operatorname{rank} \mathcal{E}_{0}$. For a general $L=\mathbb{P}^{\beta}$ in $\mathbb{P}^{m_{1}-1}, \beta=m_{1}-r=$ $\operatorname{codim}\left(Y, \mathbb{P}^{m_{1}-1}\right)$, we have $\mathbb{P}^{m_{1}-r} \cap X=\mathbb{P}^{m_{1}-r} \cap Y=\operatorname{deg}(Y)=m_{1}$. Note that $\operatorname{deg}(X)-\operatorname{codim}(X)+\left(m_{1}-r\right)-1=\left(m_{1}+m_{2}\right)-\left(m_{1}+m_{2}-1-r\right)+\left(m_{1}-r\right)-1=m_{1}$. Therefore $\mathbb{P}^{m_{1}-r}$ is a next to extremal curvilinear subspace to $X$. Note also that $\beta=m_{1}-r$ and $e=\operatorname{codim}(X)=\beta+\left(m_{2}-1\right)$

However, by Corollary $4.4, X$ has no next to extremal secant line if $\operatorname{dim}(X) \geq 3$ or $m_{2}-1 \geq 3$ for elliptic surfaces.

Remark 4.7. Let $X^{n} \subset \mathbb{P}^{n+e}$ be an $n$-dimensional scroll of degree $d$, not necessarily linearly normal, which is obtained by successive linear projections of a linearly normal scroll. Consider the ascending chain of higher secant varieties $S^{i-1}(X)$ which is the Zariski closure of the union of linear subspaces $\left\langle p_{1}, p_{2}, \ldots, p_{i}\right\rangle \simeq \mathbb{P}^{i-1}$ for $i$-points of $X$, i.e., for some $m \geq 2$,

$$
X \subset S^{1}(X) \subset S^{2}(X) \cdots \subset S^{i}(X) \subset S^{i+1}(X) \cdots \subset S^{m}(X)=\mathbb{P}^{n+e} .
$$

The image $\pi_{p}(X)$ under the projection from a point $p \in S^{2}(X) \backslash S^{1}(X)$ has a trisecant line and successive isomorphic projections starting from $p \in S^{i}(X) \backslash$ $S^{i-1}(X)$ give some $i$-secant lines to the projection image. Note that $S^{i-1}(X) \neq$ $S^{i}(X)$ if $S^{i-1}(X) \neq \mathbb{P}^{n+e}$ (see Proposition 1.3, Ch.V in [Z]). Thus a study of the position of the center of projections with respect to the higher secant varieties $S^{i}(X)$ 
should allow us to find out whether $X$ has next to extremal secant subspaces and to describe them geometrically if they exist. In addition, it would also be very helpful to compute Castelnuovo-Mumford regularity and linear syzygies $\left(N_{p}\right.$ property) for nonlinearly normal scrolls over a curve of small geometric genus if we would like to get an upper bound of degree of defining equations and consequently to find nearly extremal secant lines (cf. $[\mathrm{KP})$.

\section{ACKNOWLEDGMENTS}

This paper was started when I was visiting the Max-Planck Institut für Mathematik during the summer of 2001. The spiritual atmosphere as well as staying with Professor Jangheon Oh's family and Mr. Euisung Park in MPIM made my life there enjoyable. I thank Professors Masaaki Homma, Atsushi Noma and Masahiro Ohno for useful discussions and especially Fyodor Zak for his careful reading of my first draft and fruitful suggestion during our stay at the Korea Institute for Advanced Study (KIAS). Special thanks go to the referee for his comments and directions for further research.

\section{REFERENCES}

[AB] A. Alzati, G. M. Besana, On the k-regularity of some projective manifolds, Collect. Math. 49 (2-3) (1998), 149-171. MR2000a:14005

[ABB] A. Alzati, M. Bertolini and G. M. Besana, Projective normality of varieties of small degree, Comm. Algebra (1997), 3761-3771. MR98j:14053

[Be] M.-A. Bertin, On the regularity of varieties having an extremal secant line, J. Reine Angew. Math. 545 (2002), 167-181. MR2003h:14078

[Bu] D. C. Butler, Normal generation of vector bundles over a curve, J. Diff. Geom. 39 (1) (1994), 1-34. MR94k:14024

[D] J. D'Almeida, Courbes de l'espace projectif: Series lineaires incompletes et multisecantes, J. Reine Angew. Math. 370 (1986), 30-51. MR87k:14034

[E] D. Eisenbud, Commutative Algebra with a View Toward Algebraic Geometry, Graduate Texts in Mathematics 150, Springer-Verlag, Heidelberg, 1995. MR97a:13001

[EG] D. Eisenbud and S. Goto, Linear free resolutions and minimal multiplicity, J. Algebra 88 (1984), 89-133. MR85f:13023

[EH] D. Eisenbud and J. Harris, Varieties of minimal degree (a centennial account), Bowdoin conference in Algebraic Geometry (Brunswick, Maine 1985), Proc. Symp. Pure Math., vol. XXXXVI, part 1, Amer. Math. Soc., Providence, RI, 1987, pp. 1-13. MR89f:14042

[ES] Ph. Ellia and G. Sacchiero, Smooth surfaces in $\mathbb{P}^{4}$ ruled in conics, Algebraic Geometry (Catania, 1993/Barcelona, 1994), Lecture Notes in Pure and Appl. Math., vol. 200, Dekker, 1998, pp. 49-62. MR99j:14038

[Fu] T. Fujita, Classification theories of polarized varieties, London Mathematical Society Lecture Note Series, vol. 155, Cambridge University Press, Cambridge, 1990. MF93e:14009

[GLP] L. Gruson, R. Lazarsfeld and C. Peskine, On a theorem of Castelnuovo and the equations defining projective varieties, Inv. Math. 72 (1983), 491-506. MR85g:14033

[GP] F. Gallego and B. Purnaprajna, Normal Presentation on Elliptic Ruled Surfaces, J. Algebra 186 (1996), 597-625. MR98c:14030

[Ha] J. Harris, Algebraic Geometry, GTM 133, Springer-Verlag, 1992. MR93j:14001

[Io] P. Ionescu, Embedded projective varieties of small invariants, Algebraic Geometry (Bucharest, 1982), Lecture Notes in Math., vol. 1056, Springer-Verlag, Berlin-HeidelbergNew York, 1984. MR85m:14024

[K1] S. Kwak, Castelnuovo regularity of smooth projective varieties of dimension 3 and 4, J. Algebraic Geometry 7 (1998), 195-206. MR2000d:14043

[K2] S. Kwak, Castelnuovo-Mumford regularity for smooth threefolds in $\mathbb{P}^{5}$ and extremal examples, J. Reine Angew. Math. 509 (1999). MR2000e:14064

[K3] S. Kwak, Multisecant spaces to projective varieties, Preprint. 
[KP] S. Kwak and E. Park, Regularity and higher normality of ruled varieties over curves, Preprint.

[Mu] D. Mumford, Lectures on curves on an algebric surface, Annals of Math. Studies, vol. 59, 1966. MR 35:187

[No] A. Noma, A bound on the Castelnuovo-Mumford regularity for curves, Math. Ann. 322 (2002), 69-74. MR2002k:14046

[Oh] M. Ohno, On odd-dimensional projective manifolds with smallest secant varieties, Math. Z. 226 (3) (1997), 483-498. MR99a:14059

[SV] A. J. Sommese and A. Van de Ven, On the adjunction mapping, Math. Ann. 278 (1987), 593-603. MR88j:14011

[Z] Fyodor Zak, Tangents and secants of algebraic varieties, Translation of Math. Monographs, vol. 127, Amer. Math. Soc., 1993. MR94i:14053

Department of Mathematics, Korea Advanced Institute of Science and Technology, 373-1 Gusung-dong, Yusung-Gu, Taejeon, Korea

E-mail address: sjkwak@math.kaist.ac.kr 